\title{
Frações contínuas para a resolução da equação de Pell
}

Leomar dos Santos Veras (D)

\author{
Juscimar da Silva Araujo (D)
}

\section{Resumo}

Os números racionais e irracionais apresentam poucas pesquisas na educação básica e, quando abordados, são geralmente apresentados com foco privilegiando somente aspectos operatórios, finitos, infinitos e exatos, o que limita a abordagem e o entendimento desse importante tema no ensino da Matemática. Neste artigo mostraremos como se pode usar o conceito de frações contínuas para encontrar soluções inteiras para as equações de Pell do tipo $\mathrm{x}^{2}-d \mathrm{y}^{2}=m$, onde $m$ é um número inteiro, $d$ um número natural não nulo, e que não seja quadrado perfeito. Utilizaremos o software Geogebra para visualizar as posições das soluções.

Palavras-chave: Frações Contínuas; Equações de Pell; Soluções inteiras; Geogebra.

\section{Abstract}

The rational and irrational numbers present little research in basic education and, when they are approached, they are usually presented with a focus on privileging only operational, finite, infinite and exact aspects, which limits the approach and understanding of this important theme in the teaching of Mathematics. In this paper we will show how we can use the concept of Continuous Fractions to find whole solutions to Pell's equations of the type $\mathrm{x}^{2}-d \mathrm{y}^{2}=m$, where $m$ is an integer and $d$ is a natural number null and not a perfect square. We will use the Geogebra software to visualize the positions of the solution.

Keywords: Continuous Fractions; Pell's equations ; Whole solutions, Geogebra.

\section{Introdução}

Assunto inicialmente desenvolvido pelos gregos, as frações contínuas foram enriquecidas ao longo do desenvolvimento histórico da Matemática e retomado nos séculos XVII e XVIII. De acordo com [2], credita-se ao matemático Pietro Antonio Cataldi, nascido em Bolonha em 1548, o mérito de ter dado os primeiros passos na teoria das frações contínuas, bem como o matemático britânico William Brouncker (1620-1684) como o primeiro a investigar e usar propriedades das frações contínuas.

As frações contínuas são consideradas um dos mais belos temas da Matemática Elementar e um dos melhores instrumentos de investigação da natureza aritmética dos números irracionais. Tem aplicação na própria Matemática e em diferentes áreas do conhecimento. Suas bases teóricas foram construídas ao longo de quase três séculos, a partir das primeiras décadas do século XVII, e contaram com a contribuição de diversos matemáticos, dentre eles John Wallis(1616-1703), que foi o primeiro a utilizar o termo frações contínuas, em seu livro intitulado Opera Mathematica, de 1695 . 
Frações contínuas constituem um exemplo interessante de procedimento que é finito, quando operado sobre números racionais, e infinito, quando o número dado é irracional. A origem das frações contínuas está na Grécia, onde as frações, para efeito de comparações, eram todas escritas com numerador 1 .

Nesse artigo, apresentaremos uma outra maneira de representar números reais, a representação por frações contínuas, que sempre fornece aproximações racionais surpreendentemente boas, além de ser natural e conceitualmente simples.

O nome da equação de Pell vem do matemático suíço Leonhard Euler (1707), que em uma carta para Goldbach confundiu o nome de William Brouncker, o primeiro matemático que deu um algoritmo para resolver a equação, com a do matemático inglês John Pell (1611-1685).

A equação de Pell é um caso particular das equações diofantinas, chamadas de quadráticas quando $\mathrm{x}^{2}-\alpha \mathrm{y}^{2}=m$, com $\mathrm{x}$, y inteiros e $\alpha$ um número inteiro positivo e diferente de um quadrado perfeito.

Neste artigo, apresentamos como as Frações Contínuas podem ser uma ferramenta interessante para encontrar soluções inteiras para as equações de Pell do tipo $\mathrm{x}^{2}-d \mathrm{y}^{2}=m$, onde $m$ é um número inteiro e $d$ um número natural não nulo e que não seja quadrado perfeito.

\section{Frações Contínuas}

Seja $\alpha$ um número real. Uma expressão finita ou infinita da forma

$$
\alpha=\left[\mathrm{a}_{0}, \mathrm{a}_{1}, \mathrm{a}_{2}, \ldots\right]=\mathrm{a}_{0}+\frac{1}{\mathrm{a}_{1}+\frac{1}{\mathrm{a}_{2}+\frac{1}{\mathrm{a}_{3}+\frac{1}{\mathrm{a}_{4}+\frac{1}{\ldots}}}}}
$$

é chamada de Fração Contínua de $\alpha$, onde $\mathrm{a}_{0} \in \mathbb{Z}, \mathrm{a}_{1}, \mathrm{a}_{2}, \mathrm{a}_{3}, \ldots \geq 1$. Aqui, o símbolo [] representa o maior número inteiro menor ou igual.

Os $a_{0}, a_{1}, a_{2}, a_{3}, \ldots$ são chamados de quocientes parciais da fração contínua.

\subsection{Frações Contínuas para Números Racionais}

Nesta seção, apresentamos alguns exemplos de números racionais representados por frações contínuas, que, como o próprio nome indica, são frações que envolvem mais de um denominador ou numerador, podendo ser finita ou infinita. Trata-se de frações importantes, visto que podem ser usadas para representar números racionais, entre outras propriedades. Segue um teorema importante nessa abordagem.

Teorema 1. Todo número racional pode ser representado de duas maneiras distintas sob a forma de fração contínua finita. E toda fração contínua finita representa um número racional.

Demonstração 1. referência [1] 
Exemplo 1. $\frac{542}{234}$

$$
\begin{aligned}
& 542=234 \cdot 2+74 \Rightarrow \quad \frac{542}{234}=2+\frac{74}{234} \\
& 234=74 \cdot 3+12 \quad \Rightarrow \quad \frac{234}{74}=3+\frac{12}{74} \\
& 74=12 \cdot 6+2 \quad \Rightarrow \quad \frac{74}{12}=6+\frac{2}{12} \\
& 12=2 \cdot 6+0 \quad \Rightarrow \quad \frac{12}{2}=6 .
\end{aligned}
$$

Fazendo as substituições devidas,

$\frac{542}{234}=2+\frac{74}{234}=2+\frac{1}{\frac{234}{74}}=2+\frac{1}{3+\frac{12}{74}}=2+\frac{1}{3+\frac{1}{\frac{74}{12}}}$

$$
\begin{gathered}
=2+\frac{1}{3+\frac{1}{6+\frac{2}{12}}}=2+\frac{1}{3+\frac{1}{6+\frac{1}{\frac{12}{2}}}} \\
=2+\frac{1}{3+\frac{1}{6+\frac{1}{6}}}=[2,3,6,6] .
\end{gathered}
$$

Exemplo 2. [2, 1, 4, 2]

$$
\begin{aligned}
{[2,1,4,2]=2+\frac{1}{1+\frac{1}{4+\frac{1}{2}}}=2+\frac{1}{1+\frac{\frac{1}{9}}{\frac{2}{9}}} } & \\
& =2+\frac{1}{1+\frac{2}{9}}=2+\frac{1}{\frac{11}{9}}=2+\frac{9}{11}=\frac{22+9}{11}=\frac{31}{11} .
\end{aligned}
$$

De acordo com [4], sobre o racional $\frac{p}{q}$, temos que:

i) se $0<\mathrm{p}<\mathrm{q}$, então o primeiro quociente parcial da fração contínua é zero;

ii) se p for negativo, então o primeiro quociente parcial da fração contínua é negativo;

iii) se p > q, então o primeiro quociente parcial da fração contínua é positivo. 


\subsection{Frações Contínuas para Números Irracionais}

Os números irracionais são representados por uma sequência infinita em frações contínuas.

Exemplo 3. Calcular a expansão de $\sqrt{5}$ por frações contínuas.

Sabemos que por aproximação vale:

$$
\sqrt{5} \approx 2,2360 \ldots \quad \Rightarrow \quad\lfloor\sqrt{5}\rfloor=2,
$$

$$
\begin{gathered}
\sqrt{5}=2+\frac{1}{\mathrm{x}_{1}} \Rightarrow \sqrt{5}-2=\frac{1}{\mathrm{x}_{1}} \Rightarrow \mathrm{x}_{1}=\frac{1}{\sqrt{5}-2} \Rightarrow \mathrm{x}_{1}=\sqrt{5}+2, \\
\sqrt{\mathrm{x}_{1}} \approx 4,2360 \ldots \Rightarrow\left\lfloor\sqrt{\left.\mathrm{x}_{1}\right\rfloor}=4,\right. \\
\sqrt{5}+2=4+\frac{1}{\mathrm{x}_{2}} \Rightarrow \sqrt{5}-2=\frac{1}{\mathrm{x}_{2}} \Rightarrow \mathrm{x}_{2}=\frac{1}{\sqrt{5}-2} \Rightarrow \mathrm{x}_{2}=\sqrt{5}+2 .
\end{gathered}
$$

Portanto $\mathrm{x}_{1}=\mathrm{x}_{2}=\mathrm{x}_{3}=\ldots=\sqrt{5}+2$. Logo,

$\sqrt{5}=2+\frac{1}{\mathrm{x}_{1}}=2+\frac{1}{\sqrt{5}+2}=2+\frac{1}{4+\frac{1}{\mathrm{x}_{2}}}=2+\frac{1}{4+\frac{1}{\sqrt{5}+2}}$

$$
=2+\frac{1}{4+\frac{1}{4+\frac{1}{4+\frac{1}{\mathrm{x}_{2}}}}}=[2,4,4,4, \ldots]
$$

Assim, $[2,4,4,4, \ldots]$ é a fração contínua de $\sqrt{5}$.

Exemplo 4. Calcular a fração contínua de $\sqrt{3}$. Por valores aproximados temos que ,

$$
\sqrt{3} \approx 1,732 \ldots \Rightarrow\lfloor\sqrt{3}\rfloor=1
$$

$$
\sqrt{3}=1+\frac{1}{\mathrm{x}_{1}} \Rightarrow \sqrt{3}-1=\frac{1}{\mathrm{x}_{1}} \Rightarrow \mathrm{x}_{1}=\frac{1}{\sqrt{3}-1} \Rightarrow \mathrm{x}_{1}=\frac{\sqrt{3}+1}{2},
$$




$$
\begin{gathered}
\mathrm{x}_{1}=\frac{\sqrt{3}+1}{2} \approx 1,0366 \ldots \Rightarrow\left\lfloor\sqrt{\mathrm{x}_{1}}=1,\right. \\
\mathrm{x}_{1}=\frac{\sqrt{3}+1}{2}=1+\frac{1}{\mathrm{x}_{2}} \Rightarrow \mathrm{x}_{1}=\frac{\sqrt{3}+1}{2}-1=\frac{1}{\mathrm{x}_{2}} \Rightarrow \mathrm{x}_{2}=\sqrt{3}+1, \\
\mathrm{x}_{2}=\sqrt{3}+1 \approx 2,732 \ldots \Rightarrow\lfloor\sqrt{3}+1\rfloor=2, \\
\mathrm{x}_{2}=\sqrt{3}+1=2+\frac{1}{\mathrm{x}_{3}} \Rightarrow \mathrm{x}_{2}=\sqrt{3}-1=\frac{1}{\mathrm{x}_{3}} \Rightarrow \mathrm{x}_{3}=\frac{\sqrt{3}+1}{2} .
\end{gathered}
$$

Portanto, $x_{1}=x_{3}=x_{5} \ldots=\frac{\sqrt{3}+1}{2}$ e $x_{2}=x_{4}=x_{6}=\ldots=\sqrt{3}+1$. Logo,

$$
\begin{gathered}
\sqrt{3}=1+\frac{1}{\mathrm{x}_{1}}=1+\frac{1}{\frac{\sqrt{3}+1}{2}}=1+\frac{1}{1+\frac{1}{\mathrm{x}_{2}}}=1+\frac{1}{1+\frac{1}{\sqrt{3}+1}} \\
=1+\frac{1}{1+\frac{1}{2+\frac{1}{\mathrm{x}_{3}}}}=1+\frac{1}{1+\frac{1}{2+\frac{1}{\frac{\sqrt{3}+1}{2}}}} \\
=[1,1,2,1,2,1, \ldots] .
\end{gathered}
$$

Assim, $[1,1,2,1,2,1, \ldots]$ é a fração contínua de $\sqrt{3}$.

\section{Equações de Pell}

Examinamos, nesta seção, as soluções de equações do tipo $\mathrm{x}^{2}-\mathrm{dy}^{2}=\mathrm{m}$, onde d é um inteiro livre de quadrados e m é um inteiro qualquer. Tal equação é conhecida como Equação de Pell. Segue uma definição.

Definição 1. Considere $d$ um número natural não nulo que não seja quadrado perfeito. A equação de Pell é $\mathrm{x}^{2}-d \mathrm{y}^{2}=m$, onde $m$ é um número inteiro.

Observação 1. Aqui trataremos particularmente do caso em que $m=1$, sendo o par ordenado (x, y) uma solução inteira da equação de Pell. 
Justificativa. Supondo que $d=\mathrm{p}^{2}$ seja um quadrado perfeito (p é um inteiro) e $m=1$, então $\left(\mathrm{x}^{2}-\mathrm{p}^{2} \mathrm{y}^{2}\right)=1, \operatorname{assim}$

$$
(\mathrm{x}-\mathrm{py}) \cdot(\mathrm{x}+\mathrm{py})=1 \quad \text { ou } \quad(\mathrm{x}-\mathrm{py}) \cdot(\mathrm{x}+\mathrm{py})=-1
$$

como as soluções procuradas são inteiras, temos $(\mathrm{x}-\mathrm{py})=1 \mathrm{e}(\mathrm{x}+\mathrm{py})=1$, pois $(\mathrm{x}-\mathrm{py})=(\mathrm{x}+\mathrm{py})=1 \quad \Rightarrow \quad \mathrm{x}-\mathrm{x}=\mathrm{py}+\mathrm{py} \quad \Rightarrow \quad 0=2 \mathrm{py} \quad \Rightarrow \quad \mathrm{y}=0 \quad \Rightarrow \quad \mathrm{x}=1$.

Mas também temos que,

$(\mathrm{x}-\mathrm{py})=(\mathrm{x}+\mathrm{py})=-1 \quad \Rightarrow \quad \mathrm{x}-\mathrm{x}=\mathrm{py}+\mathrm{py} \quad \Rightarrow \quad 0=2 \mathrm{py} \quad \Rightarrow \quad \mathrm{y}=0 \quad \Rightarrow \quad \mathrm{x}=-1$.

Portanto, somente os pares $(1,0)$ e $(-1,0)$ são soluções triviais da equação de Pell.

Exemplo 5. Encontrar soluções para a equação de Pell, $\mathrm{x}^{2}-5 \mathrm{y}^{2}=1$.

Sabemos que $\sqrt{5}=[2,4,4, \ldots]$. Assim,

$$
2+\frac{1}{4}=\frac{9}{4}
$$

O par $\left(\mathrm{x}_{1}, \mathrm{y}_{1}\right)=(9,4)$ é uma solução.

Para obter outra solução,

$$
2+\frac{1}{4+\frac{1}{4+\frac{1}{4}}}=2+\frac{1}{4+\frac{1}{\frac{17}{4}}}=2+\frac{1}{4+\frac{4}{17}}=\frac{161}{72}
$$

$\mathrm{O} \operatorname{par}\left(\mathrm{x}_{2}, \mathrm{y}_{2}\right)=(161,72)$ também é solução.

Uma terceira solução:

$$
2+\frac{1}{4+\frac{1}{4+\frac{1}{4+\frac{1}{4+\frac{1}{4}} ;}}}=\frac{6748}{3033}
$$


temos que o par $\left(\mathrm{x}_{3}, \mathrm{y}_{3}\right)=(2889,1292)$ é solução.

Para uma quarta solução, seria

$$
2+\frac{1}{4+\frac{1}{4+\frac{1}{4+\frac{1}{4+\frac{1}{4+\frac{1}{4+\frac{1}{4}} .}}}}}=\frac{51841}{23184}
$$

Portanto, a equação de Pell, $\mathrm{x}^{2}-5 \mathrm{y}^{2}=1$ tem infinitas soluções inteiras.

Exemplo 6. Encontrar soluções para a equação de Pell, $\mathrm{x}^{2}-3 \mathrm{y}^{2}=1$.

Sabemos que $\sqrt{3}=[1,1,2,1,2,1, \ldots]$. Assim,

$$
1+\frac{1}{1+\frac{1}{2+\frac{1}{1}}}=\frac{7}{4}
$$

$\mathrm{O} \operatorname{par}\left(\mathrm{x}_{1}, \mathrm{y}_{1}\right)=(7,4)$ é solução.

Para obter outra solução,

$$
1+\frac{1}{1+\frac{1}{2+\frac{1}{1+\frac{1}{2+1}}}}=\frac{26}{15}
$$

Assim, o par $\left(\mathrm{x}_{2}, \mathrm{y}_{2}\right)=(26,15)$ também é solução. 


\section{Software Geogebra para visualizarmos as soluções}

Nesta seção, iremos utilizar o software Geogebra, um software de matemática dinâmica gratuito e multiplataforma, para ter uma visualização geométrica da curva de Pell, mais precisamente da equação $\mathrm{x}^{2}-5 \mathrm{y}^{2}=1$; também teremos a localização das soluções inteiras encontradas.

Na Figura 1 abaixo, temos a curva de Pell, ou seja, a curva $x^{2}-5 y^{2}=1$.

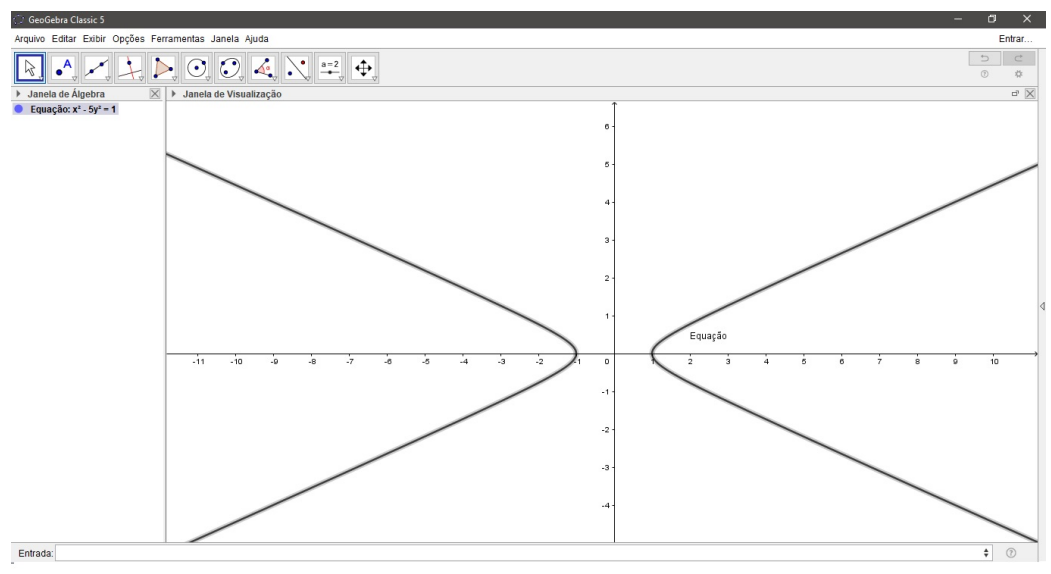

Figura 1: A curva $\mathrm{x}^{2}-5 \mathrm{y}^{2}=1$ é uma Hipérbole.

Na Figura 2 a seguir, temos as duas primeiras soluções inteiras encontradas.

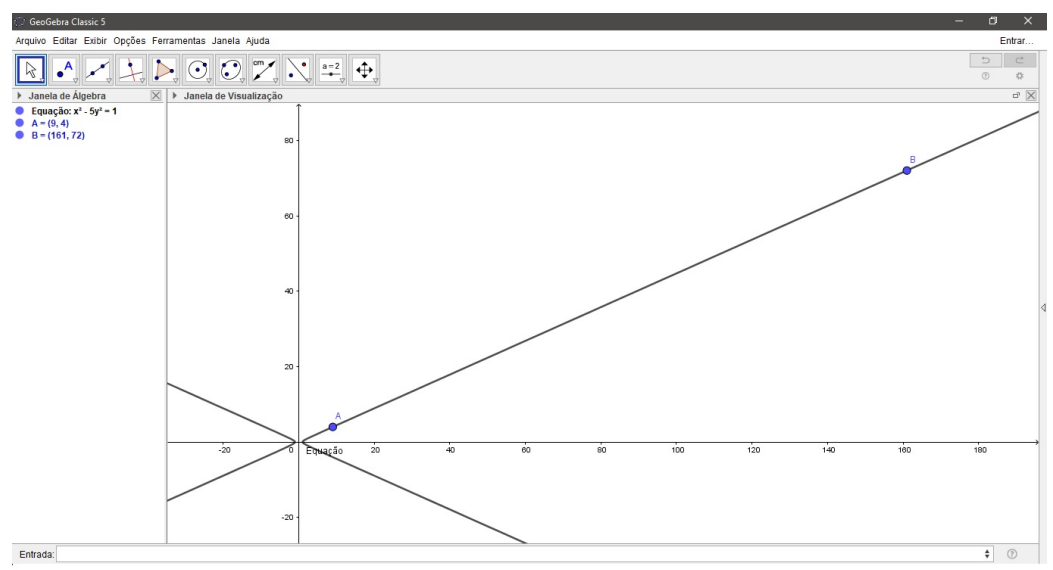

Figura 2: Localização das soluções. 
Na Figura 3 a seguir, temos representadas mais algumas soluções.

Exemplo 7. O par $(9,4)$ é solução da equação $x^{2}-5 y^{2}=1 ; \operatorname{logo}$, os pares $(-9,-4),(9,-4),(-9,4)$ também são soluções da equação de Pell.

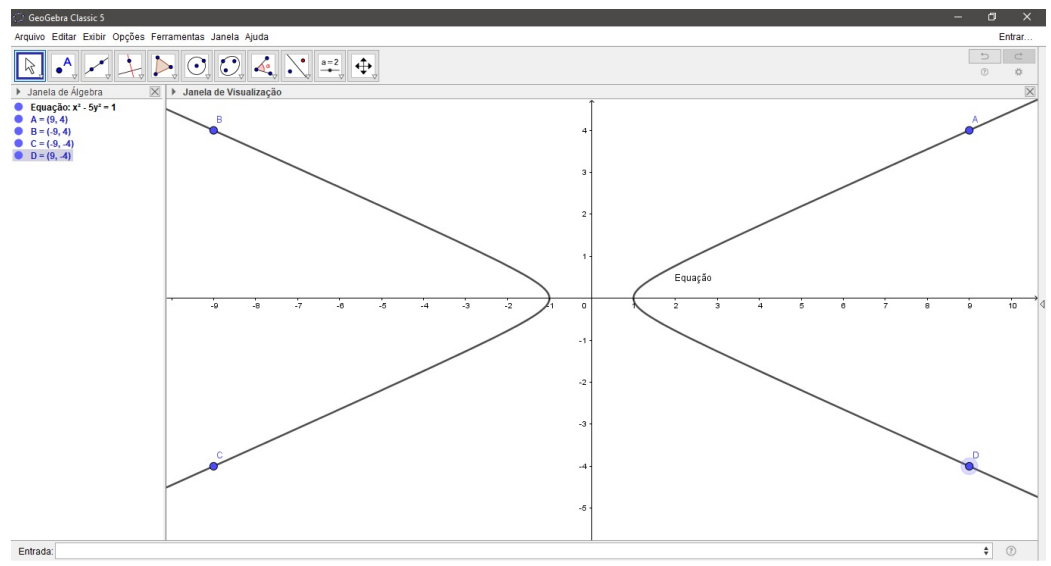

Figura 3: Outras soluções a partir de uma encontrada.

Observação 2. Sabemos que $\sqrt{5} \approx 2,23606797749978996 \ldots$; agora notemos que em

$$
\begin{gathered}
\frac{9}{4} \approx 2,25 \\
\frac{161}{72} \approx 2,23611111111 \ldots \\
\frac{2889}{1292} \approx 2,2360681114551 \ldots \\
\frac{51841}{23184} \approx 2,2360679779158 \ldots
\end{gathered}
$$

encontramos uma aproximação para $\sqrt{5}$ através das soluções da equação. 
Justificativa. Supondo que (x,y) sejam soluções para a equação $\mathrm{x}^{2}-\mathrm{dy}^{2}=1$. Assim,

$$
\begin{gathered}
\frac{x^{2}}{y^{2}}-d=\frac{1}{y^{2}} \Rightarrow\left(\frac{x}{y}\right)^{2}-d=\frac{1}{y^{2}}, \quad \text { fazendo } y \rightarrow+\infty \\
\frac{x}{y} \approx \sqrt{d} .
\end{gathered}
$$

\section{Conclusão}

Para a equação, $\mathrm{x}^{2}-5 \mathrm{y}^{2}=1$, encontramos

$$
(9,4) ;(-9,-4) ;(-9,4) ;(9,-4)
$$

$(161,72) ;(-161,-72) ;(-161,72) ;(161,-72)$

$(2889,1292) ;(-2889,-1292) ;(-2889,1292) ;(2889,-1292)$

(51841, 23184); (-51841,-23184); (-51841, 23184); (51841,-23184),

um total de 16 soluções.

O uso do conceito de Frações Contínuas é uma ótima ferramenta para encontrar soluções para as equações de Pell da forma $\mathrm{x}^{2}-d \mathrm{y}^{2}=m$, onde $d$ é um número natural não nulo que não seja quadrado perfeito e $m$ é um número inteiro.

As considerações tecidas neste artigo revelam possibilidades de abordagem das Frações Contínuas no Ensino Básico. Do ponto de vista didático, tal recurso permite ilustrar a utilização das Frações Contínuas e explorar uma série de procedimentos alternativos na representação de números.

As Frações Contínuas representam uma das possibilidades de abordar significativamente os números irracionais no ciclo básico: um número é irracional se a representação em forma de fração contínua for infinita. Caso a representação seja finita, o número é racional. Essa forma de abordagem elimina a circularidade na apresentação dos números reais, delimitando os aspectos finitos e infinitos, exatos e aproximados, discretos e contínuos, de forma simples e envolvendo conteúdos acessíveis aos alunos do ciclo básico. 


\section{Referências}

[1] Andrade, Eliana Xavier Linhares de; Bracciali, Cleonice Fátima. Frações Contínuas: algumas propriedades e aplicações. Disponível em: www.bienasbm.ufba.br/MC34.pdf.

[2] Eves, Howard. Introdução à História da Matemática. Campinas: Unicamp, 2004.

[3] Olds, C. D. Continued Fractions. Nova York, Randon House, 1963.

[4] Santos, José P. de O. Introdução à Teoria dos Números. Rio de Janeiro: Impa, 2009.

Leomar dos Santos Veras Universidade Federal do Pará $<$ leomar.santos.veras@gmail.com>

Juscimar da Silva Araujo Universidade Estadual da Região Tocantina do Maranhão $<$ juscimararaujo@uemasul.edu.br>

Ana Clara de Aguiar Sousa Universidade Estadual do Maranhão <claranaguiar@gmail.com>

Recebido: 04/11/2020

Publicado: 26/03/2021 